\title{
Modeling and identification of a small scale magnetorheological damper*
}

\author{
N. Aguirre ${ }^{\dagger}$, F. Ikhouane ${ }^{\dagger}$ \& J. Rodellar ${ }^{\dagger}$ \\ D. Wagg $^{\ddagger}$ \& S. Neild \\ $\dagger$ Department of Applied Mathematic III, Technical University of \\ Catalunya. Barcelona, Spain.E-mail: naile.aguirre@upc.edu, \\ faycal.ikhouane@upc.edu, jose.rodellar@upc.edu \\ $\ddagger$ Department of Mechanical Engineering University of Bristol Queen’s \\ Building, University Walk, Bristol, BS8 1TR, UK. E-mail: \\ david.wagg@bristol.ac.uk,simon.neild@bristol.ac.uk
}

\begin{abstract}
Magnetorheological (MR) dampers are promising vibration control devices widely used for vibration mitigation applications as they combine reliability and stability of passive systems while maintaining versatility of active devices without large power requirements. These dampers are intrinsically nonlinear, so one of the challenging aspects of applying this technology is the development of accurate models to describe their behaviour for control design and evaluation purposes. This paper deals with the parametric identification of a small scale MR damper which is modelled using the viscous + Dahl model. Experimental results show reasonably good agreement with the forces predicted by the identified model.
\end{abstract}

Keywords: MR dampers, Dahl model, Friction, Hysteresis, Identification.

\section{INTRODUCTION}

Currently, MR dampers are successfully used to improve the performance of suspension systems without the large power requirements and maintenance costs associated to active devices commonly used for similar applications.

These devices have small and large scale applications depending on their dimensions. Typically, Small MR damper forces range around $1000 \mathrm{~N}$ and have been used, among others, as shock absorbers for vehicle's and motorcycle's suspension Poynor (2001); Gravatt (2003), cars seat suspension Choi et al. (2000), vibration control in helicopters rotors F. Gandhi and Xia (2001) and prosthetic limbs J.D. Carlson and Toscano (2001). On the other hand large scale MR dampers can provide forces around $200 \mathrm{kN}$ which are suitable for civil engineering applications, among others, based isolated buildings Yoshioka et al. (2002) and bridges Chen et al. (2003).

These devices have a controllable fluid composed of suspensions of micron-sized, magnetizable particles dispersed in an appropriate carrier liquid. In the presence of a magnetic field, the iron particles form linear chains parallel to the field. These chains make it difficult for the fluid carrier to flow so that it becomes semisolid exhibiting a viscoplastic behavior Ashour et al. (1996). This transformation, illustrated in Figure 1, happens in a few milliseconds which means that MR dampers can

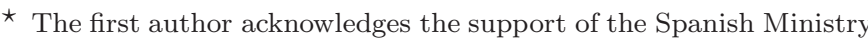
of Science and Education through FPI program and the diligent efforts of the staff at the University of Bristol at BLADE laboratory in helping to setup and conduct these experiments.
}

provide the civil engineering structures with controllable damping forces driven from an input voltage.

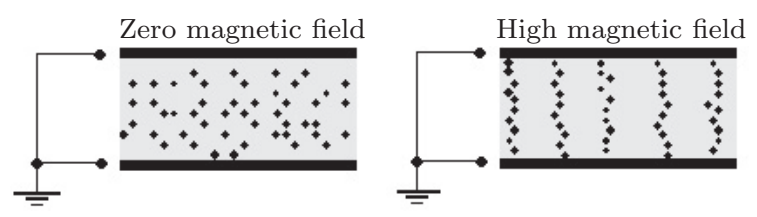

Fig. 1. MR fluid when subject to zero magnetic field and high magnetic field.

The precise modelling of these devices using the laws of physics is an arduous task and leads to complex models of limited usefulness in control applications. For this reason, alternative models have been developed by combining a physical insight with a black-box approach Savaresi et al. (2005).

One of these models that has been used to describe MR dampers is the Dahl model Dahl (1968) which consists of a first-order nonlinear differential equations that approximates experimentally observed hysteresis loops. It has been used in references Zhou et al. (2006, 2008); Şahin et al. (2010); Ikhouane and Rodellar (2007); Ikhouane and Dyke (2007); Rodriguez et al. (2009a,b) to describe the MR dampers behaviour (small and large scale).

The objective of this work is to identify a small scale MR damper which is described by the viscous + Dahl 
model. The identification methodology invoked herein is based on the input/output information once the MR damper reaches an induced limit cycle. The obtained model is validated experimentally using constant and varying voltage.

\section{EXPERIMENTAL SETUP}

An schematic of a typical small scale magnetorheological damper is shown in Figure 2. MR dampers typically consist of a hydraulic cylinder containing a moving piston submerged in a MR fluid. The magnetic field, which is perpendicular to the fluid flow, is generated by a small electromagnet in the piston head. While the fluid is primarily operating in pressure driven flow (valve) mode, the relative velocity between the two pole plates leads to a direct-shear mode as well. Hence, as the fluid flows through the fluid gap between the damper housing and the coil assembly, it is activated by the coils which are wound on the piston. Thus, the resistance to the flow of the MR fluid through the valve section of the damper provides the force mechanism.

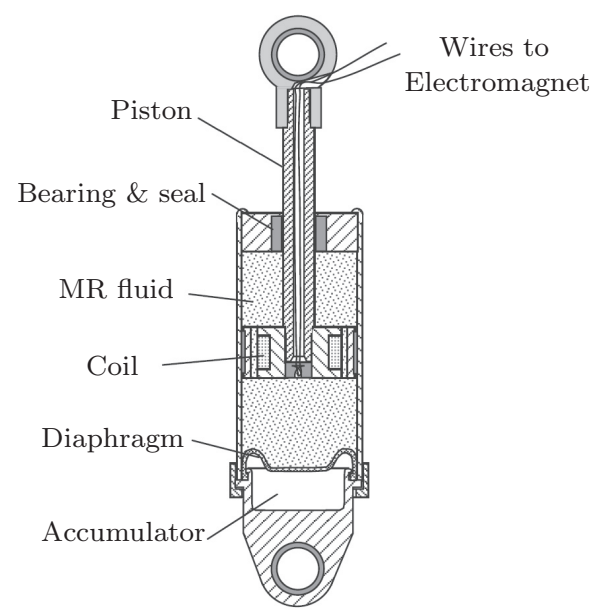

Fig. 2. Schematic of a small scale MR damper.

The MR damper used in this work is the RD-1005-3 damper shown in Figure 3 which is manufactured by Lord Corporation (http://www.lord.com/). It is $15.5 \mathrm{~cm} l o n g$, has an available stroke of $5.3 \mathrm{~cm}$. It can generate damper forces (peak to peak) of $>2224 \mathrm{~N}\left(0.05 \mathrm{~ms}^{-1}\right.$ at $\left.1 \mathrm{Amp}\right)$ and $<667 \mathrm{~N}\left(0.20 \mathrm{~ms}^{-1}\right.$ at $\left.0 \mathrm{Amp}\right)$ and can operate till a temperature of $71^{\circ} \mathrm{C}$. The damper's accumulator can accommodate a temperature change in the fluid of $27^{\circ} \mathrm{C}$. The MR damper is tested at the University of Bristol at BLADE laboratory. Figure 3 shows a picture of the damper installed for testing.

\section{MODELING AND IDENTIFICATION OF THE MR DAMPER}

\subsection{Modeling of MR dampers}

The Dahl model has been proposed independently by Dahl Dahl $(1968,1976)$ to describe frictional behaviour and by Bouc Bouc (1971) to represent hysteresis phenomena. It has been used in references Zhou et al. (2006); Ikhouane

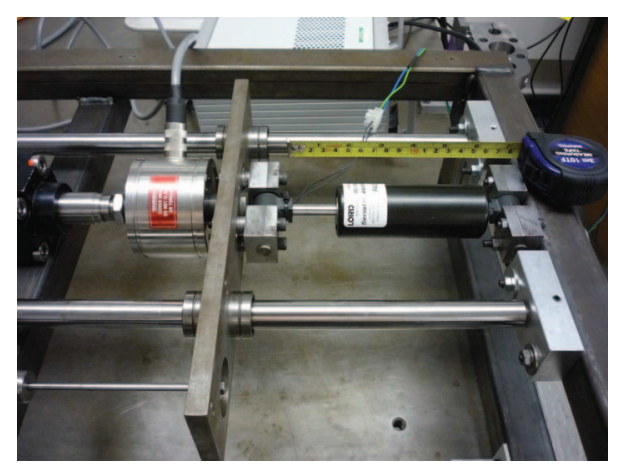

Fig. 3. Experimental set-up

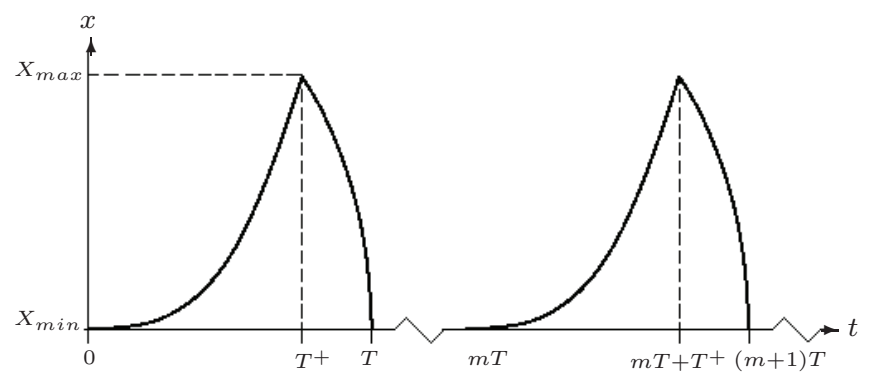

Fig. 4. Input signal $x$.

and Dyke (2007); Rodriguez et al. (2009a) to describe the MR dampers behaviour.

The model is formulated as

$$
\begin{aligned}
& F(t)=\kappa_{x}[v(t)] \dot{x}(t)+\kappa_{w}[v(t)] w(t) \\
& \dot{w}(t)=\rho[v(t)](\dot{x}(t)-|\dot{x}(t)| w(t))
\end{aligned}
$$

where $\dot{x}(t)$ denotes the damper piston velocity, $v$ the voltage input command of the amplifier, $F$ is the damping force, $w$ describes the nonlinear behaviour of the damper, and $t$ refers to time. The viscous friction coefficient $\kappa_{x}$, the dry friction coefficient $\kappa_{w}$ and the parameter $\rho$ may be voltage dependent.

\subsection{Analytic description of the forced limit cycle for the} Viscous + Dahl model

This section gives an analytical description of the hysteresis loop for the model (1)-(2). The mathematical details are given in Ikhouane and Rodellar (2005) so that this section presents only the main result. To describe analytically the hysteresis loop, the following wave $T$-periodic input signal is considered (see Figure 4). The following instrumental functions are used:

$$
\begin{aligned}
\varphi^{-}(\alpha) & =\int_{0}^{\alpha} \frac{d u}{1+u}=\ln (1+\alpha) \\
\varphi^{+}(\alpha) & =\int_{0}^{\alpha} \frac{d u}{1-u}=-\ln (1-\alpha) \\
\varphi(\alpha) & =\varphi^{-}(\alpha)+\varphi^{+}(\alpha)=\ln \left(\frac{1+\alpha}{1-\alpha}\right)
\end{aligned}
$$


where $\alpha \in(-1,1)$.

The functions $\varphi^{-}(\cdot), \varphi^{+}(\cdot)$, and $\varphi(\cdot)$ are invertible with inverses $\psi^{-}(\cdot), \psi^{+}(\cdot)$, and $\psi(\cdot)$ respectively.

$$
\begin{aligned}
\psi^{-}(\mu) & =e^{\mu}-1 \\
\psi^{+}(\mu) & =\frac{e^{\mu}-1}{e^{\mu}} \\
\psi(\mu) & =\frac{e^{\mu}-1}{e^{\mu}+1}
\end{aligned}
$$

where $\mu \in(-\infty, \infty)$.

Now, the hysteresis loop for model (1)-(2) is described by the following theorem, (Ikhouane and Rodellar, 2007, p. 47), Ikhouane and Rodellar (2005).

Theorem 1. Let $x(t)$ be a wave T-periodic input signal as depicted in Figure 4. Define the functions $\omega_{m}$ and $f_{m}$ for any non-negative integer $m$ as follows

$$
\begin{aligned}
& \omega_{m}(\tau)=w(m T+\tau) \quad \tau \in[0, T] \\
& f_{m}(\tau)=\kappa_{x} \dot{x}(\tau)+\kappa_{w} \omega_{m}(\tau) \quad \tau \in[0, T]
\end{aligned}
$$

(a) The sequence of functions $\left\{f_{m}\right\}_{m \geq 0}$ (resp. $\left\{\omega_{m}\right\}_{m \geq 0}$ ) converges uniformly on the interval $[0, T]$ to a continuous function $\bar{F}$ (resp. $\bar{w}$ ) defined as

$$
\begin{array}{r}
\bar{F}(\tau)=\kappa_{x} \dot{x}(\tau)+\kappa_{w} \bar{w}(\tau) \quad \tau \in[0, T] \\
\bar{w}(\tau)=\psi^{+}\left(\varphi^{+}\left[-\psi\left(\rho\left(I_{a}\right)\right)\right]+\rho\left(x(\tau)-X_{\min }\right)\right) \\
\tau \in\left[0, T^{+}\right] \\
\bar{w}(\tau)=-\psi^{+}\left(\varphi^{+}\left[-\psi\left(\rho\left(I_{a}\right)\right)\right]-\rho\left(x(\tau)-X_{\max }\right)\right) \\
\tau \in\left[T^{+}, T\right]
\end{array}
$$

where $I_{a}=X_{\max }-X_{\min }$

(b) For all $\tau \in[0, T]$, we have

$$
\begin{gathered}
-1<-\psi_{1,1}\left(\rho\left(X_{\max }-X_{\min }\right)\right) \leq \bar{w}(\tau) \\
\bar{w}(\tau) \leq \psi_{1,1}\left(\rho\left(X_{\max }-X_{\min }\right)\right)<1,
\end{gathered}
$$

the lower and upper bounds of $\bar{w}(\tau)$ being attained at $\tau=0$ and $\tau=T^{+}$respectively.

(See (Ikhouane and Rodellar, 2007, Sections 3.5.1 - 3.5.2), Ikhouane and Rodellar (2005) for a proof).

This result means that the output force goes asymptotically to a periodic function. The transient behavior is captured by equations (9) and (10) while the steady-state is captured by equations (11) - (13). Loading is described by equations (11) and (12) while unloading is described by equations (11) and (13). Broadly speaking, the functions $\bar{F}$ and $\bar{w}$ denote the steady-state responses of the functions $F$ and $w$ respectively.

\subsection{Identification methodology of Dahl model}

In what follows, parameters $\kappa_{x}, \kappa_{w}$ and $\rho$ are determined considering a given constant voltage. Later, this procedure is repeated for different values of the voltage to get the functions $\kappa_{x}(v), \kappa_{w}(v)$ and $\rho(v)$ referred to in Equations (1)-(2).

The identification methodology used in this paper is based on the results of Ikhouane and Rodellar (2007); Ikhouane and Dyke (2007) so that only the main steps are presented in this section. The procedure assumes the knowledge of the hysteresis loop $(\bar{F}(\tau), x(\tau))$ parameterized with the variable $\tau \in[0, T]$ which has been addressed in Theorem 1 .

To compute parameter $\kappa_{x}$ let us consider the lower and upper bounds on $\bar{w}$ (Equations (14)-(15)) to compute the values of the force at instants $\tau=0$ and $\tau=T^{+}$ respectively.

From equation 11 we have:

$$
\begin{aligned}
\bar{F}(0) & =\kappa_{x} \dot{x}(0)-\psi_{1,1}\left(\rho\left(X_{\max }-X_{\min }\right)\right) \\
\bar{F}\left(T^{+}\right) & =\kappa_{x} \dot{x}\left(T^{+}\right)+\psi_{1,1}\left(\rho\left(X_{\max }-X_{\min }\right)\right) .
\end{aligned}
$$

Thus parameter $\kappa_{x}$ can be determined as

$$
\kappa_{x}=\frac{\bar{F}(0)+\bar{F}\left(T^{+}\right)}{\dot{x}(0)+\dot{x}\left(T^{+}\right)}
$$

As explained in Ikhouane and Rodellar (2007); Ikhouane and Dyke (2007), the variable $\tau$ can be eliminated so that the functions $\bar{F}$ and $\bar{w}$ can be given as functions of $x$.

Since $\kappa_{x}$ has been computed, $\kappa_{w} \bar{w}(x)$ can be determined from Equation (1) as:

$$
\kappa_{w} \bar{w}(x)=F(x)-\kappa_{x} \dot{x} \triangleq \theta(x)
$$

Let $x_{*}$ be the value of the input such that $\theta\left(x_{*}\right)=0$. Define

$$
a=\left(\frac{d \theta(x)}{d x}\right)_{x=x_{*}}
$$

choose a design value $x_{* 1}>x_{*}$, then the parameter $\rho$ can be calculated as

$$
\rho=\frac{a-\left(\frac{d \theta(x)}{d x}\right)_{x=x_{* 1}}}{\theta\left(x_{* 1}\right)}
$$

Finally, $\kappa_{w}$ is obtained as

$$
\kappa_{w}=\frac{a}{\rho}
$$

To carry out the identification, 30 tests have been considered for each damper using different displacement input signals as detailed in Table 1 where $A=X_{\max } \mathrm{cm}$ and $f=1 / T \mathrm{~Hz}$ are the amplitude and frequency of the signal respectively. These inputs have been considered for constant input voltage from $0 \mathrm{~V}$ to $1.8 \mathrm{~V}$.

\subsection{Sensitivity of the parameter $\kappa_{x}$}

Figure 5 gives the response of the MR damper to a wave $T$-periodic input signal with $f=0.6 \mathrm{~Hz}, A=0.8 \mathrm{~cm}$ and a constant input voltage of $v=1.8 \mathrm{~V}$. In the Forcevelocity plot it can be observed that the term of the force associated to the viscous friction $\kappa_{x} \dot{x}(t)$ is smaller than the one associated to the dry friction $\kappa_{w} \bar{w}(t)$ 
Table 1. Experiments

\begin{tabular}{|c|c|c|c|c|c|c|c|c|}
\hline$A(\mathrm{~cm}) \backslash f(\mathrm{~Hz})$ & 0.3 & 0.5 & 0.6 & 1.0 & 1.3 & 1.5 & 2.0 & 2.4 \\
\hline 0.25 & & $\mathrm{x}$ & & $\mathrm{x}$ & & $\mathrm{x}$ & $\mathrm{x}$ & $\mathrm{x}$ \\
\hline 0.3 & & & & & $\mathrm{x}$ & $\mathrm{x}$ & & \\
\hline 0.5 & $\mathrm{x}$ & $\mathrm{x}$ & & $\mathrm{x}$ & & $\mathrm{x}$ & $\mathrm{x}$ & $\mathrm{x}$ \\
\hline 0.8 & & & $\mathrm{x}$ & & & & & \\
\hline 1.0 & & $\mathrm{x}$ & $\mathrm{x}$ & & & $\mathrm{x}$ & $\mathrm{x}$ & $\mathrm{x}$ \\
\hline
\end{tabular}

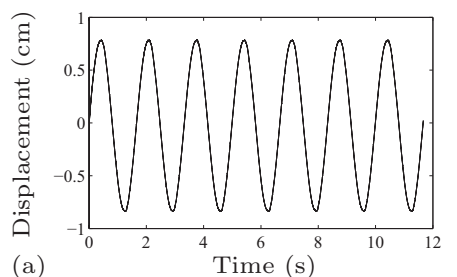

(a)
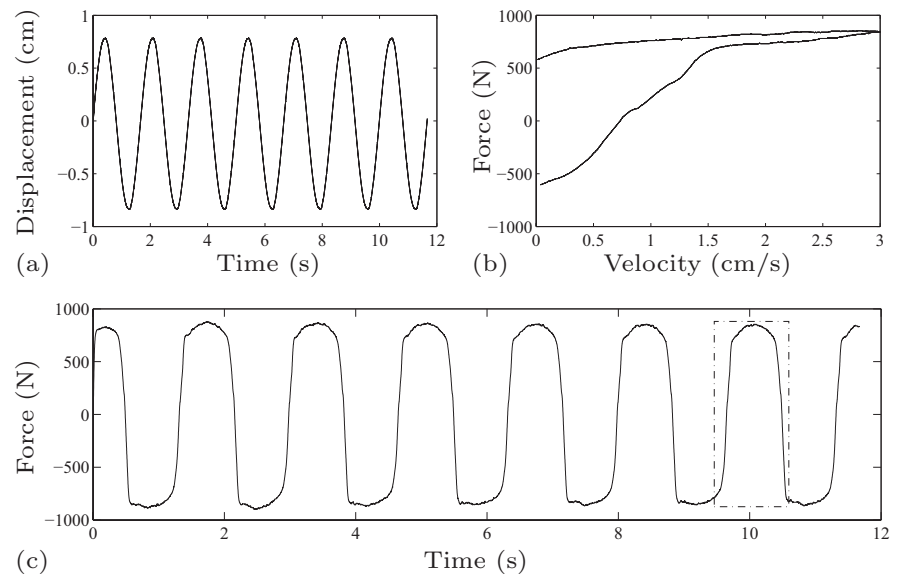

(c)

Time (s)

Fig. 5. (a) Input signal $x$ as a function of time. (b) Forcevelocity plot for $9.6 \leq t \leq 10.4 \mathrm{~s}$ (see dashed box on the Force-time plot). (c) Force-time plot response.

$$
\begin{gathered}
\max \left(\kappa_{x} \dot{x}(t)\right)=175 \mathrm{~N} \\
t \in[9.6,10.4 s] \\
\max \left(\kappa_{w} \bar{w}(t)\right)=680 \mathrm{~N} \\
t \in[9.6,10.4 s]
\end{gathered}
$$

The consequence of this observation on the identification method is now analyzed. In equation (16), let $\Delta h$ be the absolute value of the highest uncertainty on $h$. Then we have

$$
\begin{aligned}
\frac{\Delta \kappa_{x}}{\kappa_{x}} & =\frac{\Delta\left(\bar{F}(0)+\bar{F}\left(T^{+}\right)\right)}{\left|\bar{F}(0)+\bar{F}\left(T^{+}\right)\right|}+\frac{\Delta\left(\dot{x}(0)+\dot{x}\left(T^{+}\right)\right)}{\left|\dot{x}(0)+\dot{x}\left(T^{+}\right)\right|} \\
& =\frac{\Delta(\bar{F}(0))+\Delta\left(\bar{F}\left(T^{+}\right)\right)}{\left|\bar{F}(0)+\bar{F}\left(T^{+}\right)\right|}+\frac{\Delta(x(0))+\Delta\left(\dot{x}\left(T^{+}\right)\right)}{\left|\dot{x}(0)+\dot{x}\left(T^{+}\right)\right|}
\end{aligned}
$$

Let us consider that in equation (1) the viscous friction term $\kappa_{x} \dot{x}(t)$ is very small with respect to the dry friction term $\kappa_{w} w(t)$. In this case, the restoring force of the damper is given by $F(t) \simeq \kappa_{w} w(t)$. By Theorem 3 (Ikhouane and Rodellar, 2007, p. 47),Ikhouane and Rodellar (2005)) we have $\bar{F}(0)=-\psi_{1,1}\left(\rho\left(X_{\max }-X_{\min }\right)\right)$ and $\bar{F}\left(T^{+}\right)=$ $\psi_{1,1}\left(\rho\left(X_{\max }-X_{\min }\right)\right)$, so that $\bar{F}(0)+F\left(T^{+}\right) \simeq 0$. This equality along with equation (21) shows that the relative error on the parameter $\kappa_{x}$ is high if the viscous friction is much smaller than the dry friction. In our case, experimental values $\bar{F}(0)=-615 \mathrm{~N}$ and $\bar{F}\left(T^{+}\right)=561 \mathrm{~N}$ show that $\bar{F}(0)+$ $\bar{F}\left(T^{+}\right)=54 \mathrm{~N}$ is very small.

Then, equation (16) may lead to a large relative error to determine the parameter $\kappa_{x}$. In next section, it is proposed an alternative method for the determination of this parameter.

\subsection{Modified identification methodology}

In (Ikhouane and Rodellar, 2007, Eq. 4.93), Ikhouane and Dyke (2007) it is shown that the hysteresis loop has a plastic region when the displacement is large enough. This region is characterized by $\bar{w}(\tau) \simeq 1$. In this case, equation (1) becomes

$$
F(x)=\kappa_{x}[v(t)] \dot{x}(t)+\kappa_{w}[v(t)]
$$

This equation is linear in $\dot{x}(t)$ so that the constants $\kappa_{x}$ and $\kappa_{w}$ can be determined by a linear regression for each constant voltage and not by equations (16) and (20). Indeed, in Figure 5 (top right), it is observed that the force versus velocity plot presents a linear part for velocities $1.50 \leq \dot{x}(t) \leq 2.93\left(\mathrm{cms}^{-1}\right)$ which means that our assumption has thus been validated experimentally so that equation (22) can be used to identify parameters $\kappa_{x}$ and $\kappa_{w}$.

Finally, the parameter $\rho$ is computed from equation (20) as:

$$
\rho=\frac{a}{\kappa_{w}}
$$

3.6 Experimental results for experiment with input signal displacement of $f=0.6 \mathrm{~Hz}$ and $A=0.8 \mathrm{~cm}$ and constant voltage $v=1.8 \mathrm{~V}$

In this section we are computing the parameters $\kappa_{x}$, $\kappa_{w}, \rho$, based on the experimental data obtained for the experiment referred to in Figure 5. For identification purpose, only the loading part of one cycle in the steady state is needed (see Figure $5 b$ ).

The first step of the identification process of Section 3.5 is the linear regression of Equation (22). This is done in the force velocity plot of Figure $5 \mathrm{~b}$ for velocities $1.50 \leq$ $\dot{x}(t) \leq 2.93\left(\mathrm{cms}^{-1}\right)$. It is found that $\kappa_{x}=67.4\left(\mathrm{~N} \mathrm{~s} \mathrm{~cm}^{-1}\right)$ and $\bar{\kappa}_{w}=644 \mathrm{~N}$ which lead to the linear approximation shown in Figure 6a.

Then, function $\theta(x)$ is obtained from Equation (17), it is found that $x_{*}=-0.81 \mathrm{~cm}$ (see Figure $6 \mathrm{~b}$ ) and Equation (18) gives $a=1.227 \times 10^{4}\left(\mathrm{Ncm}^{-1}\right)$. Finally, the value $\rho=19\left(\mathrm{~cm}^{-1}\right)$ is obtained from Equation (23).
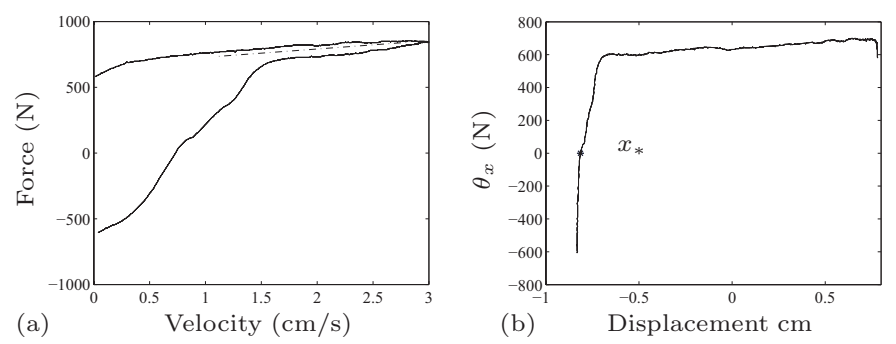

Fig. 6. (a) (solid) force-velocity plot, (dashed) linear regression of Equation (22). (b) function $\theta(x), x_{*}$ verifies $\theta\left(x_{*}\right)=0$.

\subsection{Identification results}

Table 2 shows some results of the identification methodology detailed in the previous section along with the signal 
input parameters.

Table 2. Identification results

\begin{tabular}{|cccccc|}
\hline $\begin{array}{c}\kappa_{x} \\
\left(\mathrm{~N} \mathrm{~s} \mathrm{~cm}^{-1}\right)\end{array}$ & $\begin{array}{c}\kappa_{w} \\
(\mathrm{~N})\end{array}$ & $\begin{array}{c}\rho \\
\left(\mathrm{cm}^{-1}\right)\end{array}$ & $\begin{array}{c}A \\
(\mathrm{~cm})\end{array}$ & $\begin{array}{c}f \\
(\mathrm{~Hz})\end{array}$ & $\begin{array}{c}v \\
(\mathrm{~V})\end{array}$ \\
\hline 6.47 & 68.8 & 75.3 & 0.25 & 1.5 & 0.0 \\
13 & 71.9 & 63.8 & 0.50 & 0.5 & 0.0 \\
10.3 & 43.1 & 108 & 0.25 & 0.5 & 0.0 \\
8.54 & 37.7 & 90.6 & 0.30 & 1.3 & 0.0 \\
7.55 & 64.4 & 29.7 & 0.50 & 1.5 & 0.0 \\
177 & 642 & 26.1 & 0.50 & 0.3 & 1.8 \\
94.9 & 668 & 18.2 & 0.50 & 0.5 & 1.8 \\
30.3 & 748 & 14.7 & 0.50 & 1.0 & 1.8 \\
67.4 & 644 & 19 & 0.80 & 0.6 & 1.8 \\
45.8 & 701 & 15.3 & 1.00 & 0.5 & 1.8 \\
\hline
\end{tabular}

\section{VALIDATION RESULTS}

From the identification results of the tests referred to in Table 1 , it is found that $\rho$ is a voltage-independent parameter so that it is taken as the mean value $\bar{\rho}=$ $47.95\left(\mathrm{~cm}^{-1}\right)$. Parameters $\kappa_{x}$, and $\kappa_{w}$ are voltage dependent parameters in the form

$$
\begin{aligned}
& \kappa_{x}(v)=\kappa_{x_{a}}+\kappa_{x_{b}} v \\
& \kappa_{w}(v)=\kappa_{w_{a}}+\kappa_{w_{b}} v
\end{aligned}
$$

where $\kappa_{x_{a}}=9.78\left(\mathrm{Nscm}^{-1}\right), \kappa_{x_{b}}=40.75\left(\mathrm{~N} \mathrm{scm}^{-1} \mathrm{~V}^{-1}\right)$, $\kappa_{w_{a}}=60.11 \mathrm{~N}$ and $\kappa_{w_{b}}=344.78\left(\mathrm{NV}^{-1}\right)$.

Then, the MR damper model is obtained as:

$$
\begin{aligned}
F(t) & =\left[\kappa_{x_{a}}+\kappa_{x_{b}} v(t)\right] \dot{x}(t)+\left[\kappa_{w_{a}}+\kappa_{w_{b}} v(t)\right] w(t) \\
\dot{w}(t) & =\bar{\rho}(\dot{x}(t)-|\dot{x}(t)| w(t)) \\
w(0) & =\frac{F(0)-\left[\kappa_{x_{a}}+\kappa_{x_{b}} v(0)\right] \dot{x}(0)}{\kappa_{w_{a}}+\kappa_{w_{b}} v(0)}
\end{aligned}
$$

The experimental force is denoted $F_{e}$ and the force calculated by model (26) is denoted $F$. The discrepancy between $F_{e}$ and $F$ is measured by the $L^{1}$ norm as

where

$$
\varepsilon=\frac{\left\|F_{e}-F\right\|_{1}}{\left\|F_{e}\right\|_{1}}
$$

$$
\|f\|_{1}=\int_{0}^{T_{e}}|f(t)| d t .
$$

$T_{e}$ and $f$ are the time duration and force time function for each experiment.

\subsection{Validation results with constant voltage}

The validation of the model was done using the same inputs referred to in Table 1 . Figure 7 shows the comparison between predicted and experimental data for the experiments with $f=1.5 \mathrm{~Hz}, A=0.5 \mathrm{~cm}$ and $v=0 \mathrm{~V}$ and $f=1 \mathrm{~Hz}, A=0.25 \mathrm{~cm}$ and $v=1.8 \mathrm{~V}$. The relative errors are $\varepsilon=18.3 \%$ and $\varepsilon=12.1 \%$ respectively.

Validation of the all tests leads to the average relative error of $22 \%$.

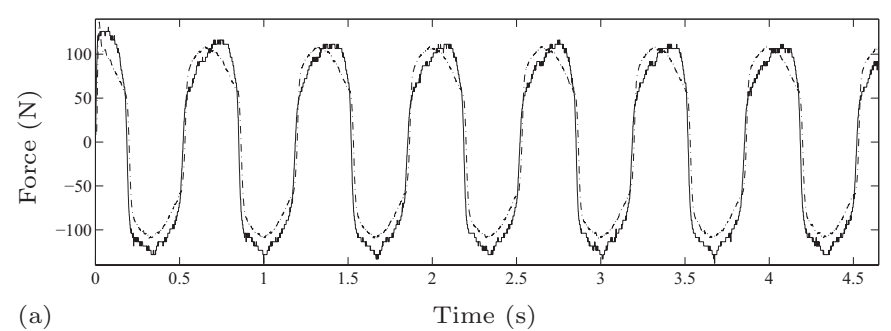

(a)

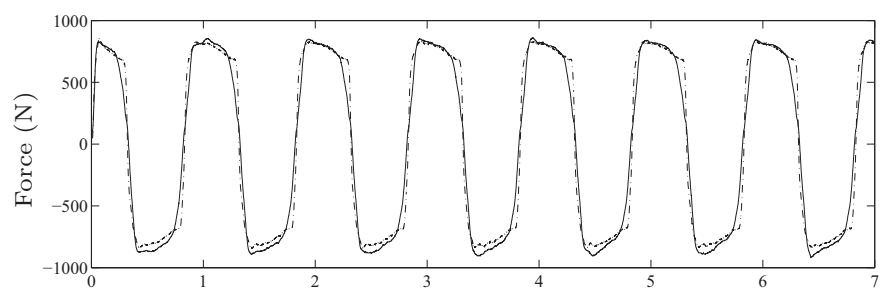

(b)

Time (s)

Fig. 7. Comparison between predicted (dashed line) and experimental force (solid line). (a) $f=1.5 \mathrm{~Hz}$, $A=0.5 \mathrm{~cm}$ and $v=0 \mathrm{~V}$. (b) $f=1 \mathrm{~Hz}, A=0.25 \mathrm{~cm}$ and $v=1.8 \mathrm{~V}$.

\subsection{Validation results with varying voltage}

In control applications, voltage (or current) is the control variable Yoshioka et al. (2002); Dyke et al. (1996); Sahasrabudhe and Nagarajaiah (2005); Casciati et al. (2006); Jansen and Dyke (2000). This is why it is important to validate the model with a varying voltage input along with a varying displacement. We choose random signals for both inputs with a low frequency content as happens in civil engineering applications. Figure 8 shows the comparison between the experimental and predicted forces. The relative error is of $22 \%$ which is reasonable for control applications.
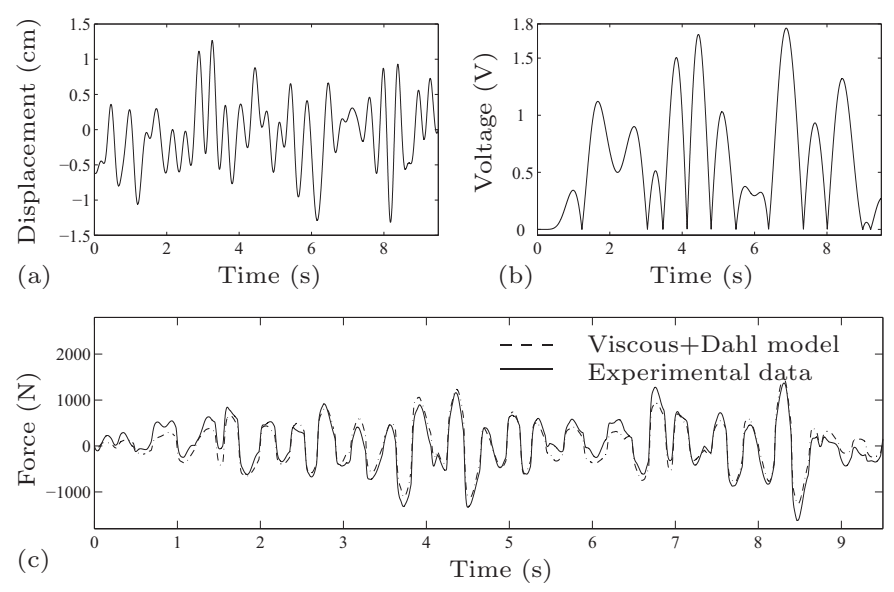

Fig. 8. Model output and experimental data. (a) Random input displacement. (b) Random input voltage. (c) Comparison between predicted and experimental force. 


\section{CONCLUSION}

This paper has dealt with the modeling and identification of a small scale MR damper. The model that has been chosen is simpler than the Bouc-Wen hysteresis model commonly used for the description of these devices. The MR damper parameters have been computed from an identification methodology and the obtained model has been validated experimentally for constant and varying voltage cases. It has been observed that the model describes very well the behaviour of the MR damper.

\section{ACKNOWLEDGEMENTS}

This work is supported by grants DPI2005-08668-C03-01 and DPI2008-06463-C02-01. The first author acknowledges the support of the Spanish Ministry of Science and Education through FPI program and the diligent efforts of the staff at the University of Bristol at BLADE laboratory in helping to setup and conduct these experiments.

\section{REFERENCES}

Ashour, O., Rogers, C.A., and Kordonsky, W. (1996). Magnetorheological fuids: materials, characterization and devices. J. Intel. Mat. Syst. Str., 7, 123-130.

Bouc, R. (1971). Modle mathmatique d'hystrsis (a mathematical model for hysteresis). Acustica, 21, 1625.

Casciati, F., Magonette, G., and Marazzi, F. (2006). Technology of semiactive devices and applications in vibration mitigation. John Wiley \& Sons Inc.

Chen, Z.Q., Wang, X.Y., Ko, J.M., N, Y.Q., Spencer, B.F., and Yang, G. (2003). Mr damping system on dongting lake cable-stayed bridge. In Proceedings of SPIE, volume 5057, 229-235.

Choi, S.B., Nam, M.H., and Lee, B.K. (2000). Nonlinear modelling of an electro-rheological vibration damper. $J$. Intel. Mat. Syst. Str., 11, 936-944.

Şahin, I., Engin, T., and Ş. Çeşmeci (2010). Comparison of some existing parametric models for magnetorheological fluid dampers. J. Smart Mater. Struct., 19.

Dahl, P.R. (1968). A solid friction model. Technical report, The Aerospace Corporation, El Secundo, CA.

Dahl, P.R. (1976). Solid friction damping of mechanical vibrations. AIAA J., 14, 1675-1682.

Dyke, S.J., Spencer, J.B.F., Sain, M.K., and Carlson, J.D. (1996). Modeling and control of magnetorheological dampers for seismic response reduction. J. Smart Mater. Struct., 5, 565-575.

F. Gandhi, K.W.W. and Xia, L. (2001). Magnetorheological fluid damper feedback linearization control for helicopter rotor application. J. Smart Mater. Struct., 10 .

Gravatt, J.W. (2003). Magnetorheological dampers for super-sport motorcycle applications. Ph.D. thesis, Virginia Polytechnic Institute and State University.

Ikhouane, F. and Dyke, S.J. (2007). Modeling and identifcation of a shear mode magnetorheological damper. Smart Materials and Structures, 16, 605-616.

Ikhouane, F. and Rodellar, J. (2005). On the hysteretic Bouc-Wen model. Part I: forced limit cycle characterization. Nonlinear Dyn., 42, 63-78.
Ikhouane, F. and Rodellar, J. (2007). Systems with Hysteresis: Analysis, Identification and Control Using the Bouc-Wen Model. Chichester, UK: Wiley.

Jansen, L.M. and Dyke, S.J. (2000). Semi-active control strategies for the MR damper: comparative study. J. Eng. Mech., ASCE, 126, 795-803.

J.D. Carlson, W.M. and Toscano, J. (2001). Smart prosthe tics based on magnetorheological fluids. In Proceedings of SPIE, volume 4332, 308-316.

Poynor, J. (2001). Innovative designs for magnetorheological dampers. Master's thesis, Virginia Polytechnic Institute and State University.

Rodriguez, A., Ikhouane, F., Rodellar, J., and Luo, N. (2009a). Modelling and identification of a small-scale magnetorheological damper. J. Smart Mater. Struct., 20, 825-835.

Rodriguez, A., Iwata, N., Ikhouane, F., and Rodellar, J. (2009b). Model identification of a large-scale magnetorheological fluid damper. J. Smart Mater. Struct., 18, Article number 015010.

Sahasrabudhe, S. and Nagarajaiah, S. (2005). Semi-active control of sliding isolated bridges using MR dampers: an experimental and numerical study. J. Earthquake Engng. Struct. Dyn., 34, 965-983.

Savaresi, S.M., Bittanti, S., and Montiglio, M. (2005). Identification of semi-physical and blackbox non-linear models: The case of MR dampers for vehicles control. Automatica, 41, 113-127.

Yoshioka, H., Ramallo, J.C., and Jr, B.F.S. (2002). Smart base isolation strategies employing magnetorheological dampers. J. Eng. Mech., 128, 540-551.

Zhou, Q., Nielsen, S.R.K., and Qu, W.L. (2006). Semiactive control of three-dimensional vibrations of an inclined sag cable with magnetorheological dampers. $J$. Sound. Vib., 296, 1-22.

Zhou, Q., Nielsen, S.R.K., and Qu, W.L. (2008). Semiactive control of shallow cables with magnetorheological dampers under harmonic axial support motion. $J$. Sound. Vib., 311, 683-706. 